\title{
Special Issue Introduction: The Place of Self-Harm in Adolescent Development
}

\author{
Roger J. R. Levesque
}

Published online: 24 December 2009

(C) Springer Science+Business Media, LLC 2009

The study of adolescence appropriately includes fascinating research agendas that remind us of how adolescents are not as troubled and troubling as many would have us believe. Indeed, the prior editor of this journal, Daniel Offer, spearheaded much of the rethinking of negative perceptions of adolescents' experiences and development (see Offer, 2007; Levesque, 2007). Yet, there is no denying that some youth considerably suffer and that many youth inflict harm on themselves. We have known for quite some time that the most obvious forms of self-harm, such as cutting, mutilation, and injury, are present in approximately $15 \%$ of community samples of adolescents (see, e.g., Laye-Gindhu and Schonert-Reichl 2005). Somewhat surprisingly, researchers studying healthy adolescent development have not investigated the roots of adolescents' self harm as much as one might have expected, and even more striking has been the failure to consider the breadth of self-destructive behaviors. During the past 5 years, for example, this journal only has published five articles directly related to self-harm, with the articles ranging from a focus on suicidality (Hüsler et al. 2005), nonsuicidal self-harm (Laye-Gindhu and SchonertReichl 2005), friends' suicides (Liu, 2006) and factors contributing to suicidal ideation (Sun \& Hui, 2007) and to suicidal behavior (Sigfusdottir et al. 2008). A quick look at leading developmental journals focusing on adolescents but not associated with clinical, counseling or medical associations reveals that each either has published none (Journal of Adolescent Research and Journal of Early Adolescence) or only two (Journal of Research on Adolescence and Youth and Society) over the same time period. Although it is important not to loose sight of the reality that many youth

R. J. R. Levesque ( $\square)$

Indiana University, Bloomington, IN, USA

e-mail: rlevesqu@indiana.edu transition relatively well into and out of adolescence, it seems equally important not to neglect the harms that adolescents suffer on their own volition. Equally important is the need to realize that the reasons adolescents may harm themselves and put themselves at risk rest in relationships that they have with others and in the wide variety of sociocultural forces that likely shape their entire development.

The relative failure of those of us studying adolescent development to address this area of research led to the development of this special issue. This special issue invited manuscripts focusing on the place of self-harm in adolescent development. Submitted manuscripts could examine a wide variety of issues relating to deliberate self-harm (e.g., self-mutilation and suicide) as well as non-deliberate selfinjury (such as engagement in high risk activities and injuries). The special issue's goal was to both deepen and expand our understanding of what constitutes self-harm as well as its causes. Given the breadth of what self-harm could encompass, efforts were made to invite researchers who typically have not published in adolescent development journals. The response was absolutely fantastic.

The articles deliver on this special issue's goals. The call for papers attracted a high number of exceptionally strong manuscripts and eventually resulted in three journal issues rather than one. The breadth of disciplinary frameworks that authors bring to our special issue is impressive, as it ranges from developmental psychology, psychiatry, medicine, neuroscience, epidemiology, criminology, education, social work, clinical psychology, and sociology to public health. As a result, the articles bring together a strong group of studies that range in their strengths and foci, with some providing longitudinal analyses, others addressing methodological challenges (e.g., measurement and sampling issues), with others addressing important ecological contexts (e.g., family, partner, peer, school, neighborhoods, 
and/or cultural and ethnic factors), and with others focusing on individual, psychological factors. Importantly, many articles address influential precursors of self-harm, its developmental consequences, as well as its prevention and possible interventions; and some articles ask us to stretch our thinking about what actually should be considered selfharm. Given the relative lack of research focusing on "selfharm" published in journals dedicated to adolescent development, it is hoped that this compilation of studies will help reinvigorate this part of our field and encourage others to study often hidden and ignored topics that nevertheless have important consequences for adolescents, their healthy development, and their place in society.

\section{References}

Hüsler, G., Blakeney, R., \& Werlen, E. (2005). Adolescent risk: The co-occurrence of illness, suicidality, and substance use. Journal of Youth and Adolescence, 34, 547-557.
Laye-Gindhu, A., \& Schonert-Reichl, K. A. (2005). Nonsuicidal selfharm among community adolescents: Understanding the "whats" and "whys" of self-harm. Journal of Youth and Adolescence, 34, $447-457$.

Levesque, R. J. R. (2007). Reflections on a legendary contribution to the empirical study of adolescence. Journal of Youth and Adolescence, 36, 1-4.

Liu, R. X. (2006). Vulnerability to friends' suicide influence: the moderating effects of gender and adolescent depression. Journal of Youth and Adolescence, 35, 454-464.

Offer, D. (2007). An academic journey. Journal of Youth and Adolescence, 36, 9-21.

Sigfusdottir, I. D., Asgeirsdottir, B. B., Gudjonsson, G. H., \& Sigurdsson, J. F. (2008). A model of sexual abuse's effects on suicidal behavior and delinquency: The role of emotions as mediating factors. Journal of Youth and Adolescence, 37, 699712.

Sun, R. C. F., \& Hui, E. K. P. (2007). Psychosocial factors contributing to adolescent suicidal ideation. Journal of Youth and Adolescence, 36, 775-786. 